\title{
The MKKK62-MKK3-MAPK7/14 module negatively regulates seed dormancy in rice
}

\author{
Xingxue Mao ${ }^{1,2+}$, Jianjun Zhang ${ }^{3 \dagger}$, Wuge Liu ${ }^{1,2 \dagger}$, Shijuan Yan ${ }^{4}$, Qing Liu ${ }^{1,2}$, Hua Fu ${ }^{1,2}$, Junliang Zhao ${ }^{1,2}$, \\ Wenjie Huang ${ }^{4}$, Jingfang Dong ${ }^{1,2}$, Shaohong Zhang ${ }^{1,2}$, Tifeng Yang ${ }^{1,2}$, Wu Yang ${ }^{1,2}$, Bin Liu ${ }^{1,2^{*}}$ and Feng Wang ${ }^{1,2^{*}}$
}

\begin{abstract}
Background: Seed dormancy directly affects the phenotype of pre-harvest sprouting, and ultimately affects the quality and yield of rice seeds. Although many genes controlling seed dormancy have been cloned from cereals, the regulatory mechanisms controlling this process are complex, and much remains unknown. The MAPK cascade is involved in many signal transduction pathways. Recently, MKK3 has been reported to be involved in the regulation of seed dormancy, but its mechanism of action is unclear.
\end{abstract}

Results: We found that MKKK62-overexpressing rice lines (OE) lost seed dormancy. Further analyses showed that the abscisic acid (ABA) sensitivity of OE lines was decreased. In yeast two-hybrid experiments, MKKK62 interacted with MKK3, and MKK3 interacted with MAPK7 and MAPK14. Knock-out experiments confirmed that MKK3, MAPK7, and MAPK14 were involved in the regulation of seed dormancy. The OE lines showed decreased transcript levels of OSMFT, a homolog of a gene that controls seed dormancy in wheat. The up-regulation of OsMFT in MKK3-knockout lines (OE/mkk3) and MAPK7/14-knockout lines (OE/mapk7/mapk14) indicated that the MKKK62-MKK3-MAPK7/ MAPK14 system controlled seed dormancy by regulating the transcription of OsMFT.

Conclusion: Our results showed that MKKK62 negatively controls seed dormancy in rice, and that during the germination stage and the late stage of seed maturation, ABA sensitivity and OsMFT transcription are negatively controlled by MKKK62. Our results have clarified the entire MAPK cascade controlling seed dormancy in rice. Together, these results indicate that protein modification by phosphorylation plays a key role in controlling seed dormancy.

Keywords: Rice (Oryza sativa L.), Pre-harvest sprouting, Dormancy, MAPK cascade

\section{Background}

Pre-harvest sprouting (PHS) reduces the production levels of rice (Oryza sativa L.), wheat, maize, barley, sorghum, and soybean crops worldwide, leading to substantial agricultural losses. Therefore, it has attracted the interest of researchers in diverse fields (Fang and Chu 2008; Li et al. 2004; Simsek et al. 2014). To date, however, PHS remains a serious problem in agricultural production.

Seed dormancy is determined by genetic and environmental factors (Finkelstein et al. 2008; Footitt et al. 2011; Footitt et al. 2013; Nakamura et al. 2011). In the wild, seed dormancy is vital for plants to avoid adverse

\footnotetext{
*Correspondence: Ibgz1009@163.com; fwang1631@163.com

${ }^{+}$Xingxue Mao, Jianjun Zhang and Wuge Liu contributed equally to this work.

${ }^{1}$ Guangdong Academy of Agricultural Sciences, Rice Research Institute,

Guangzhou 510640, China

Full list of author information is available at the end of the article
}

environments, as it ensures that seeds germinate only when environmental conditions are suitable for plant growth and development. It is an adaptive trait and the result of natural selection in wild plants (Finkelstein et al. 2008). During the long history of domestication, seed dormancy has been selectively abandoned to achieve uniform germination and higher germination percentages (Shu et al. 2016; Sugimoto et al. 2010). To reduce PHS, appropriate seed dormancy should be re-introduced.

Many hormones have been reported to regulate seed dormancy, with gibberellins (GAs) and abscisic acid (ABA) being the two most important ones (Footitt et al. 2011; Shu et al. 2016; Vaistij et al. 2013). Transcriptomic analyses of seed development and germination have shown that GAs and ABA play important roles in regulating gene expression for seed dormancy (Sreenivasulu 
et al. 2008; Wan et al. 2008). During seed development, ABA has a role in suppressing germination. After breaking dormancy, the ABA content decreases and the GAs content increases to promote germination (Shu et al. 2016). The synthesis, degradation, and signal transduction of ABA and GAs play important roles in the conversion between dormancy and germination (Finkelstein et al. 2008; Graeber et al. 2012; Graeber et al. 2014; Holdsworth et al. 2008; Shu et al. 2016)

Most of the dormancy-related genes that have been cloned from cereals so far are involved in the synthesis or signal transduction of GAs or ABA (Jin et al. 2018; Shu et al. 2016). Sdr4 was the first seed dormancy gene cloned from rice, and its transcription was shown to be controlled by OsVP1 (a homolog of the ABA-responsive transcription factor ABI3). In Arabidopsis, AtMFT was shown to regulate ABA sensitivity and in wheat, TaMFT was found to positively regulate seed dormancy (Chono et al. 2015; Li et al. 2014; Nakamura et al. 2011; Tao et al. 2014; Xi et al. 2010). Mutations of qSD1-2, which controls GA synthesis, led to decreased GA levels and plant height, and enhanced seed dormancy (Ye et al. 2015a). Although a number of genes involved in seed dormancy have been identified, our knowledge about this process is still limited. To facilitate crop breeding, more details of the mechanisms controlling seed germination need to be dissected.

The MAPK cascade is an evolutionarily conserved signaling module that plays vital roles in eukaryotes, including animals, yeast, and plants. The plant MAPK cascade system is more complex than those of animals because plants cannot escape from harsh environmental conditions. The MAPK cascade converts extracellular stimuli into intracellular signals and plays important roles in various signal transduction pathways (Colcombet and Hirt 2008). Many studies have shown that MAPK cascades mediate the signal transduction of hormones such as ABA, jasmonic acid, and ethylene in response to environmental signals (Choi et al. 2017; Danquah et al. 2015; Li et al. 2017; Matsuoka et al. 2015; Ye et al. 2015b; Yoo et al. 2008). The traditional MAPK cascade system is composed of three kinds of protein kinases: MKKK, MKK, and MAPK. Each kinase in the MAPK cascade module is sequentially activated by a relay of phosphorylation events. The activated MAPK phosphorylates downstream proteins to modify their activities and transmit the corresponding signal. Several entire cascades have been clarified. For example, the MKKK1-MKK2-MAPK4/6 module mediates cold and salt stress tolerance (Teige et al. 2004); and YODA-MKK4/5-MAPK3/6 regulates stomatal development (Lampard et al. 2009). The MKKKs start the cascade, and are activated or transcriptionally regulated by environmental or developmental signals. It is particularly important to understand the functionality of MKKKs. Recently, many reports have shown that the MAPK cascade participates in the regulation of seed germination. The MKK1-MAPK6 module was shown to transmit the ABA signal and control the seed germination process by regulating the expression of CAT1 and the glucose-induced ABA level (Xing et al. 2008, 2009). Map-based cloning studies have shown that $M K K 3$ controls seed dormancy in barley and wheat (Nakamura et al. 2016; Torada et al. 2016). Two genes in the Raf subfamily of MKKK genes, Raf10 and Raf11, were found to positively regulate seed dormancy in Arabidopsis (Lee et al. 2015).

In this study, we found that the overexpression of MKKK62 in rice led to the loss of seed dormancy and decreased ABA sensitivity. Protein interaction analyses confirmed interactions between downstream members of the MAPK cascade, and their function in dormancy regulation was verified by knockout experiments. These analyses have revealed the details of the MAPK cascade that controls seed dormancy in rice.

\section{Results \\ Overexpression of $M K K K 62$ in rice results in loss of seed dormancy}

In rice, there are $75 M K K K \mathrm{~s}$, eight $M K K \mathrm{~s}$, and 15 MAPKs (Group. M 2002; Hamel et al. 2006; Rao et al. 2010). Relatively few studies have focused on these kinases, especially the MKKKs. It was reported that the expression level of MKKK62 was greatly affected by the external environment (Rao et al. 2010). To examine the expression pattern of $M K K K 62$, we conducted real-time PCR using total RNA extracted from the shoot, leaf, and seed. The transcript level of MKKK62 was high at the late stage of seed maturation, suggesting that MKKK62 affected certain characteristics of mature seeds (Additional file 1: Figure S1). To study its function, we cloned MKKK62 from the cultivar ZH11, a Japonica rice variety. The encoded protein was identical to XP_015639022.1. We then overexpressed MKKK62 in ZH11 under the control of the maize ubiquitin promoter (Ubi::MKKK62). Three homozygous $\mathrm{T}_{2}$ MKKK62-overexpressing (OE) transgenic lines (OE1, OE2, OE3) were selected for characterization.

The OEs showed a PHS phenotype. To confirm the phenotype at 30 days after heading (DAH), we collected the panicles and tested the seed germination percentage immediately. At 3 days after imbibition (DAI), all the seeds from homozygous OEs germinated, while few seeds of wild type (WT) germinated (Fig. 1a). To test the germination ability at the seed-filling stage, we harvested seeds at different times, kept them under germination conditions for 2 days, and then calculated the germination percentage. At 2 DAI, several seeds collected from 
a

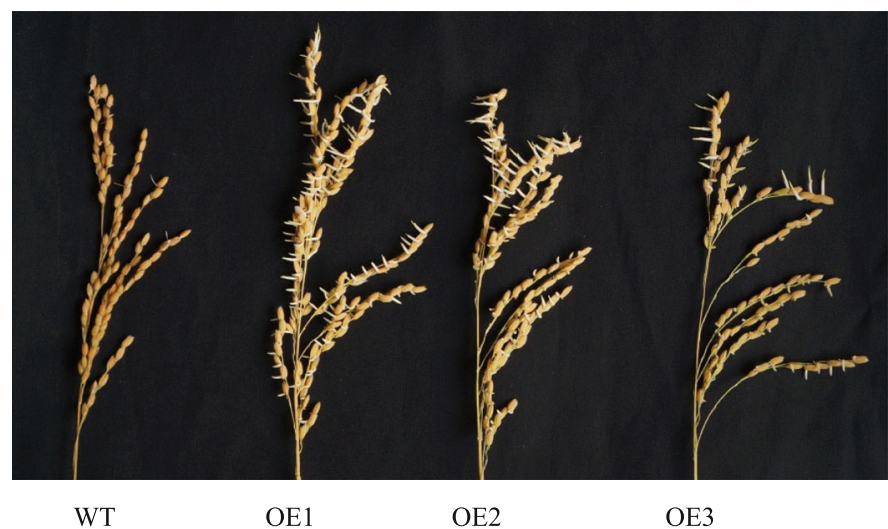

b

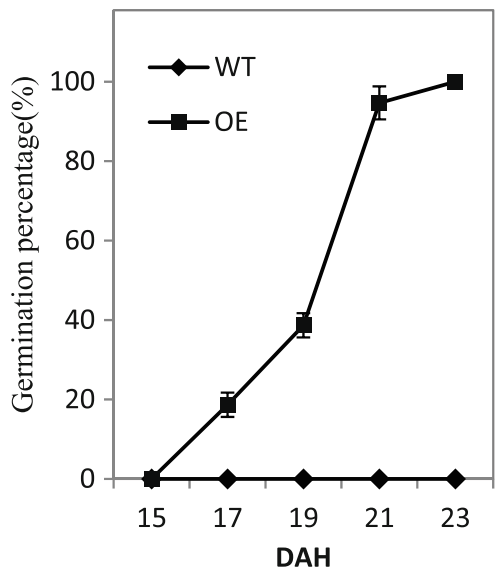

c

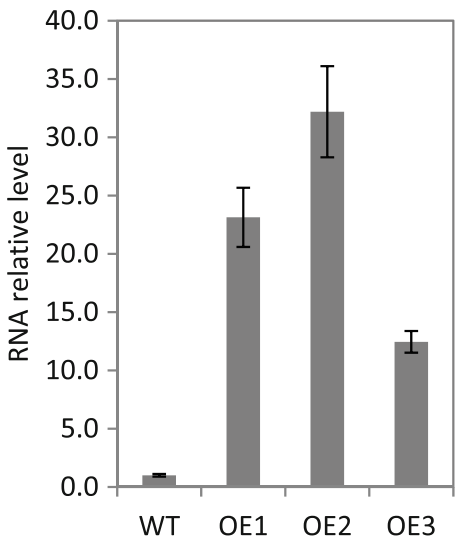

Fig. 1 Overexpression of MKKK62 in ZH11 resulting in PHS. a, Germination phenotype of WT and OE panicles. At 30 DAH, panicles of OE1, OE2, OE3, and WT were sampled for germination tests. Seeds from OE lines germinated at 3 DAI. b, Germination percentage of seeds from OE panicles sampled at indicated times. Seeds were harvested at indicated times from OE plants for germination test. Germination percentage was calculated at 2 DAl. Values represent the mean \pm SD of three biological replicates. C, Relative expression level of MKKK62 in seeds of MKKK62-overexpression (OE) lines at 23 DAH. At 23 DAH, gene transcript levels in seeds of OE1, OE2, OE3, and WT were analyzed by real-time PCR

the OEs at 18 DAH germinated, all seeds collected from the OEs at 23 DAH germinated, but none of the WT seeds germinated (Fig. 1b, Additional file 2: Figure S2). The real-time PCR assay results showed that the transcript levels of MKKK62 were higher in seeds of the three OE lines than in ZH11 seeds at 23 DAH (Fig. 1c).To investigate the effect of MKKK62 on seed dormancy, we investigated the germination characteristics of seeds from heterozygous OEs. At 23 DAH, we collected the panicles and tested the seed germination percentage. At 2 DAI, the germinated seeds were counted and then planted. Analyses of DNA extracted from the leaves of the seedlings confirmed that all of them contained the marker gene, and so all were transgenic lines. The non-germinating seeds were harvested and cultivated on 1/2 Murashige \& Skoog medium, and then analyzed to determine whether they contained the marker gene. None of the plants derived from non-germinated seeds were transgenic lines (Table 1, Additional file 3: Figure S3). This result also confirmed that the overexpression of MKKK62 led to the loss of seed dormancy.

\section{$A B A$ and $G A s$ contents in seeds}

Both $A B A$ and GAs are important hormones in the regulation of seed dormancy. To determine whether the

Table 1 Seed germination of progeny of three heterozygous OE lines

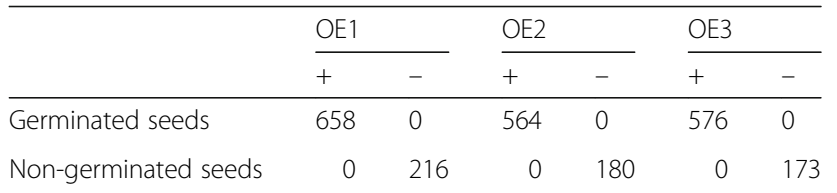

Note: Five panicles were harvested at $23 \mathrm{DAH}$ from each of three heterozygous $\mathrm{OE}$ lines for the germination test. Numbers of seeds germinated/ non-germinated after 2 days are shown. Plus sign, transgenic progeny; minus sign, non-transgenic progeny 
PHS phenotype of OEs was due to changes in the contents of ABA or GAs, we quantified these hormones in the seeds of WT and the three OEs by LC-MS/MS. The contents of $\mathrm{GA}_{3}$ and $\mathrm{GA}_{4}$ were similar in the OEs and WT, while the ABA content was significantly higher in the OEs than in WT (Fig. 2).

\section{Decrease in $A B A$ sensitivity during germination of $\mathrm{OE}$ seeds}

In plants, ABA positively regulates dormancy. To evaluate the effect of ABA to suppress the germination of $\mathrm{OE}$ seeds, we treated $\mathrm{OE}$ seeds with different concentrations of $\mathrm{ABA}$ at $23 \mathrm{DAH}$. According to this result, $10 \mu \mathrm{M}$ ABA was suitable to show the suppression effect of ABA on seed germination of OE (Additional file 4: Figure S4). Germination and especially post-germination growth are usually inhibited by ABA (Lopez-Molina et al. 2001). To evaluate the sensitivity of post-germination growth to ABA, identically sprouted seeds of OE and WT were selected and placed on agar containing ABA. After 3 days, the shoots of $\mathrm{OE}$ were significantly longer than those of WT in the mock treatment $(P<0.05)$ (Fig. 3a, and b). In the ABA treatment, the shoots and roots of the OE lines were significantly longer than those of WT $(P<0.01)$. Compared with the mock treatment, relative root and shoot elongation was much greater in OE than in WT (Fig. 3a, and b). This result indicated that ABA inhibited shoot and root growth much more strongly in WT than in OE. Together, these results showed that ABA sensitivity was decreased in the OE lines.

\section{Identification of protein interactions}

The MAPK module usually consists of MKKK-MKK-MAPK. Usually, MKKK62 will interact with and activate downstream MKKs, and the activated MKKs will interact with and activate the downstream

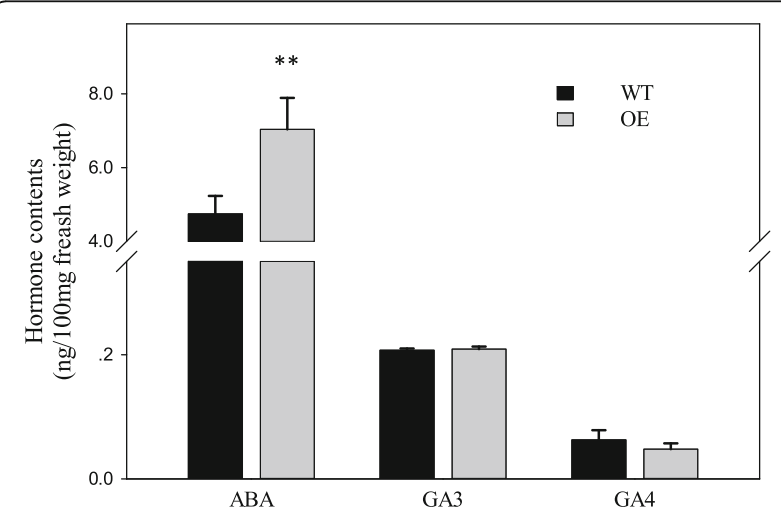

Fig. $2 A B A$ and GAs contents in seeds of OE and WT lines. At 23 $D A H$, seeds from three OE lines and WT were harvested and stored in liquid nitrogen until analysis. Values represent the mean \pm SD of at least three replicates. Student's t-tests were used to generate $P$ values
MAPKs. To explore these relationships, we performed $\mathrm{Y} 2 \mathrm{H}$ experiments. The coding region of MKKK62 was cloned and inserted into a bait vector (pGBKT7). We amplified the eight rice $M K K$ genes using seed cDNA as the template and the primer pairs listed in Additional file 8: Table S2. Only seven MKKs were successfully amplified and cloned into the prey vector (pGADT7). No autoactivation activity was detected in yeast (data not shown). Every MKKK62-MKK pair was co-transformed into yeast cells, and the obtained colonies were cultured on double-dropout medium. The co-transformed cells were then streaked onto quadruple-dropout medium containing $200 \mathrm{ng} / \mathrm{mL}$ Aureobasidin A (AbA). According to the growth on quadruple-dropout medium, MKKK62 interacted with MKK3 and MKK10-2. We used a similar method to screen for proteins interacting with MKK3. We found that MKK3 interacted with two of the 15 MAPK proteins, MAPK7 and MAPK14 (Fig. 4a). None of the MAPK proteins interacted with MKK10-2. To further test the interaction, we performed pull-down experiments. The pull-down assay using E. coli-produced recombinant proteins confirmed the following interactions: MKKK62-MKK3, MKKK62-MKK10-2, MKK3-MAPK7, and MKK3-MAPK14(Fig. 4b, c, d, and e). Based on the $\mathrm{Y} 2 \mathrm{H}$ and pull-down results, MKK3, MKK10-2, MAPK7, and MAPK14 were identified as candidate downstream proteins of MKKK62.

\section{Knockout of downstream genes increased seed dormancy of OE lines}

To explore the roles of downstream proteins of MKKK62 in regulating dormancy, we constructed CRISPER vectors to knockout certain downstream genes. The amino acid sequences of MAPK7 and MAPK14 were similar, suggesting that they may have redundant functions. Therefore, we constructed the vector MB-MAPK7/14 to knock out both genes simultaneously. After Agrobacterium-mediated genetic transformation, we obtained 10 independent transgenic lines containing MB-MKK3 and three independent lines containing MB-MAPK7/14.

Using DNA from leaves of $\mathrm{T}_{2}$-generation plants as the template, a fragment containing the target site was amplified and sequenced. Progenies with frameshift mutations were selected for dormancy analysis. Tables 2 and 3 show the target sites and sequences of $M K K 3, M A P K 7$, and MAPK14 in various knockout progenies.

In the late cropping season in 2017, we selected the frame-shift mutants in the $\mathrm{T}_{2}$ generation for seed dormancy analysis. Seeds were harvested at $23 \mathrm{DAH}$ for the germination test, and the germination percentage was calculated at 2 DAI. The seeds from homozygous plants of $\mathrm{OE} / m k k 3$ and $\mathrm{OE} / m a p k 7 / m a p k 14$ did not germinate, 
a

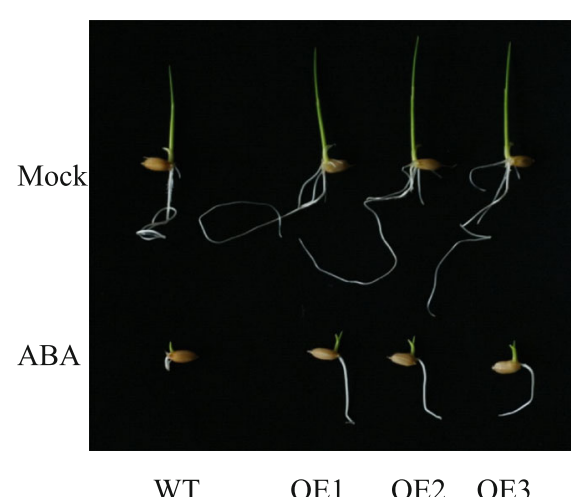

b

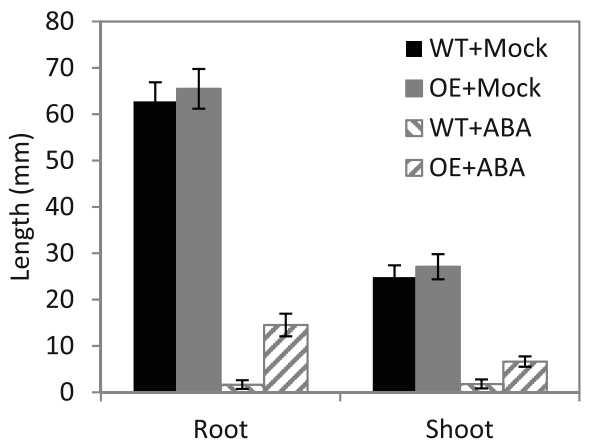

Fig. 3 Decreased ABA sensitivity in OE lines. a, Growth performance of OE lines and WT on $1 \%(w / v)$ agar without or with $10 \mu M$ ABA. In mock treatment, agar contained equal amount of solvent. b, Length of shoot and root of OE lines after 3 days of treatment. Shoot and root lengths were measured after 3 days of culture in the light at $30^{\circ} \mathrm{C}$.Values represent the mean $\pm \mathrm{SD}$ of three OE lines (ten replicates/line)

but most seeds from heterozygous plants of OE/ $m k k 3(\mathrm{H})$ and $\mathrm{OE} / \operatorname{mapk} 7(\mathrm{P}) / \operatorname{mapk} 14(\mathrm{H})$ germinated, and all seeds from homozygous plants of OE/mapk7 germinated (Fig. 5a). Seeds harvested from homozygous plants of $\mathrm{OE} / m k k 3$ and $\mathrm{OE} /$ mapk7/mapk14 at $30 \mathrm{DAH}$ did not germinate, even at 5 DAI (Fig. 5b). After 1 month of storage at $37^{\circ} \mathrm{C}$ to break dormancy, we tested the germination percentage of seeds from homozygous plants of $\mathrm{OE} / m k k 3$ and $\mathrm{OE} / m a p k 7 / m a p k 14$. After breaking dormancy the germination percentage of OE/mapk7(P)/ mapk14(P) increased greatly and above $60 \%$ of seeds from $\mathrm{OE} / \mathrm{mkk} 3(\mathrm{P})$ also germinated at $8 \mathrm{DAI}$ (Fig. 5c). It indicated that the knockout of MKK3 or MAPK7/14 is nonlethal to seeds. These results revealed that the mutation of $M K K 3$ or $M A P K 7 / 14 /$ could stop the PHS phenotype induced by the overexpression of MKKK62.

\section{Expression analysis of genes related to dormancy}

Genes that have been reported to regulate seed dormancy in cereals include Sdr4, OsDOG1-likeOsVp1, TaMFT, and qSD7-1 (Bentsink et al. 2006; Gu et al. 2011; Hattori et al. 1994; Nakamura et al. 2011; Sugimoto et al. 2010). To investigate whether MKKK62 affects the expression of these genes, we collected seeds from the OE lines at $23 \mathrm{DAH}$ and analyzed gene transcript levels by real-time PCR. The transcript level of OsMFT in seeds was significantly lower in the OE lines than in WT (Fig. 6). There was no significant difference in the transcript levels of Sdr4, OsDOG1-like, OsVp1, and $q S D 7-1$ between WT and OE (data not shown). Further analyses showed that the transcript levels of OsMFT were much higher in homozygous $\mathrm{OE} / m k k 3(\mathrm{P})$ and $\mathrm{OE} /$ mapk7(P)/mapk14(P) seeds than in WT seeds (Fig. 6). These results indicated that the MAPK cascade controls the expression of OsMFT in rice seeds.

\section{Discussion}

With the increasing frequency of climatic anomalies, high temperatures and long-term rain often occur during the rice-harvesting period, especially for early rice crops in tropical and subtropical regions. Therefore, to increase rice production, it is very important to enhance seed dormancy and avoid yield losses from PHS.

\section{MKKK62 control seed dormancy}

Rice $M K K K \mathrm{~s}$ can be classified into three main subfamilies; the MEKK, ZIK, and Raf subfamilies. The MEKK subfamily is most similar to animal MEKKs, which phosphorylate and activate the MEK protein. The MEKK subfamily in rice has 22 members, including MKKK62 (Rao et al. 2010). We detected high transcript levels of $M K K K 62$ at the late stage of seed maturation, suggesting that $M K K K 62$ affected seed traits (Additional file 1: Figure S1). Subsequent experiments showed that overexpression of $M K K K 62$ in $\mathrm{ZH} 11$ resulted in the loss of seed dormancy (Fig. 1a, b, Additional file 2: Figure S2). The seed dormancy of N22 (an Indica rice variety) is very strong (Wan et al. 2006), but overexpression of MKKK62 in N22 led to the loss of seed dormancy (Additional file 5: Figure S5). We also overexpressed LOC103652526 (the maize homolog of MKKK62) in rice (ZH11) and the transgenic progeny showed a similar phenotype to that of the OE lines (data not shown). These results indicated that regulation of seed dormancy by $M K K K 62$ is a common phenomenon among cereals.

Seed dormancy is determined by the seed embryo, endosperm, testa, and husk (Finch-Savage and Leubner-Metzger 2006; Ye et al. 2015a). The genotypes of testa and husk are identical to that of the mother plant, and the embryo genotype was the same as the genotype of the seedling grown from each seed. Endosperm genotype is closely related to embryo genotype. 


\section{a}

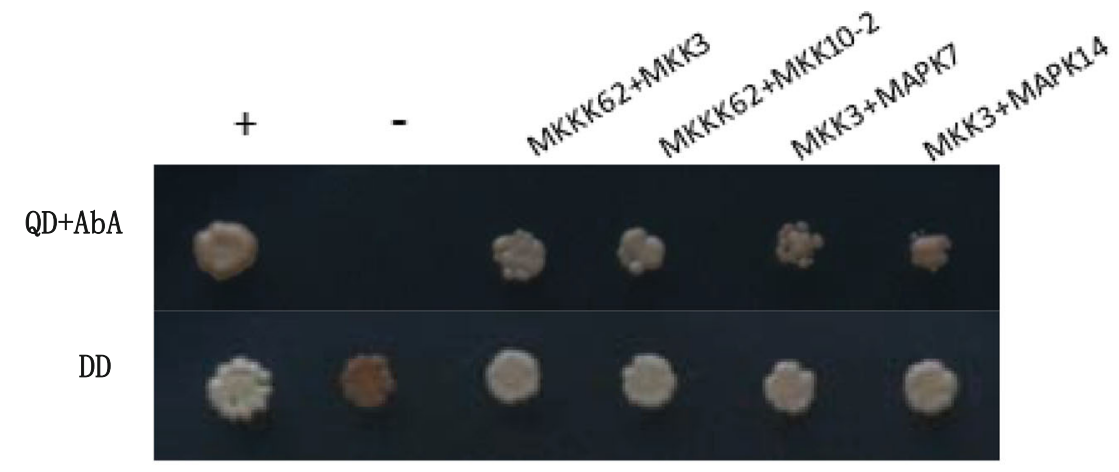

b

c

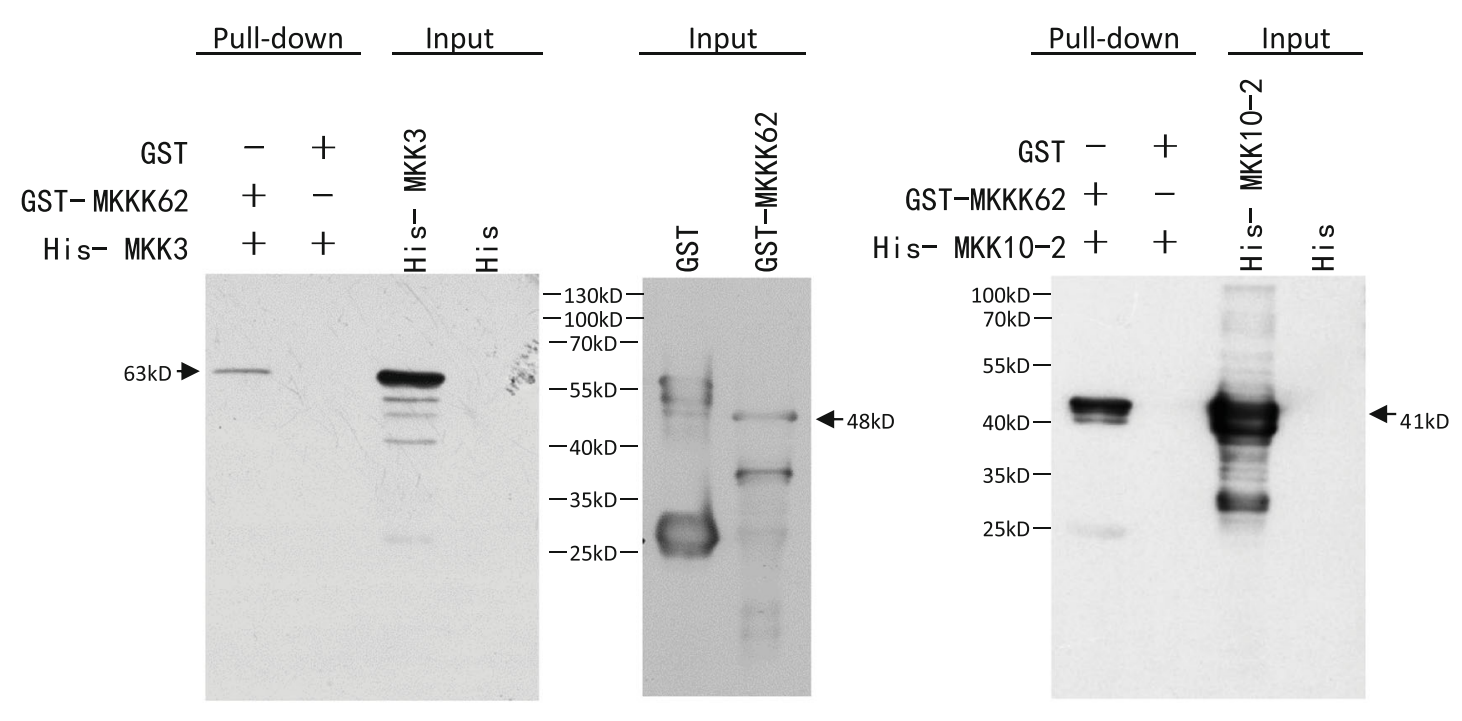

d

e

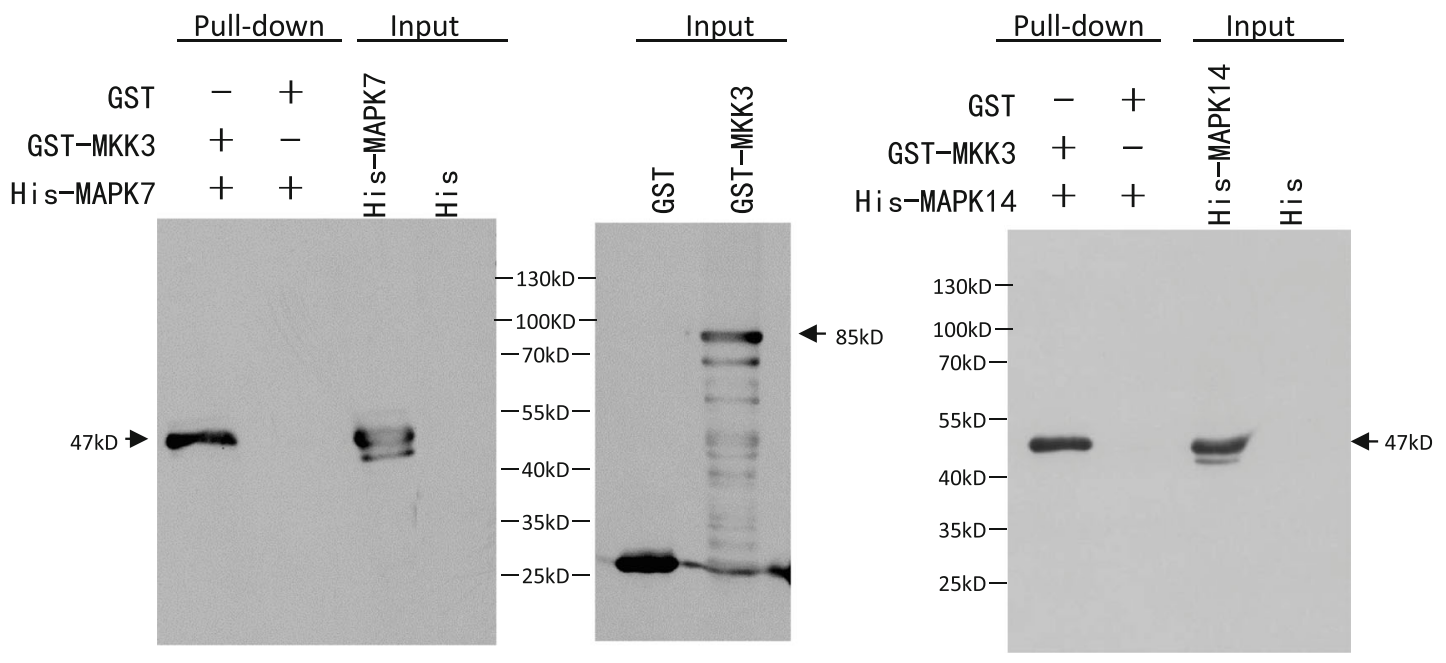


Fig. 4 Interactions between indicated proteins. a, Interactions between indicated proteins were investigated by Y2H. Plus sign, positive control; minus sign, negative control; QD + AbA, quadruple-dropout medium lacking Ade, His, Leu and Trp containing 200 ng/mL AbA; DD, doubledropout medium lacking Leu and Trp. b, c, d, and e, pull-down results of four pairs of proteins: MKKK62-MKK3, MKKK62-MKK10-2, MKK3-MAPK7, and MKK3-MAPK14, respectively. Binding of GST- and His-fusion proteins was tested by immunoblotting using anti-His after pull-down with GSTfusion protein. GST was used as negative control. Expression of fusion proteins was detected using antibody to the tag. "Input" indicated the detection result of the fusion proteins with anti-His or anti-GST antibody; "Pull-down" indicated the detection result of His-fusion protein binding to GST-fusion protein with anti-His antibody. Arrow indicates the band of the target protein

Statistical analyses of seed dormancy in the progenies of heterozygous OE lines showed that all the plants that grew from germinating seeds were transgenic positive and those that grew from dormant seeds were transgenic negative. Thus, the overexpression of $M K K K 62$ in the embryo and endosperm significantly decreased seed dormancy levels, while the overexpression of MKKK62 in the testa and husk did not (Table 1, Additional file 3: Figure S3).

We also knocked out MKKK62 in ZH11 by gene editing technology and obtained three independent lines (Additional file 6: Table S1). However, the germination efficiency (percentage of germinating seeds) of the knockout lines was not obviously decreased (Additional file 7: Figure S6). Our explanation for this result is as follows: In Arabidopsis, at least five MKKKs (AtMKKK14/15/16/17/18) are able to activate AtMKK3 (Choi et al. 2017; Matsuoka et al. 2015). In rice, there are at least eight genes showing strong similarity to MKKK62 (Rao et al. 2010), and six of them are transcribed in seeds (Sato et al. 2013). We speculated that these six genes can functionally complement the mutation of MKKK62. AtMKKK14/15/16/17/18 in Arabidopsis also belong to the MEKK subfamily. According to the phylogenetic tree, their predicted amino acid sequences show strong similarity to that of MKKK62 (Rao et al. 2010). However, there were no significant changes in the seed dormancy of Arabidopsis overexpressing AtMK KK14/15/16/17/18 (Choi et al. 2017; Matsuoka et al. 2015). In Arabidopsis, ABA was shown to positively regulate the expression of $A t M K K K 18$ and promote the phosphorylation of the downstream proteins, AtMAP K1/2/7/14 (Danquah et al. 2015; Leonhardt et al. 2004). Overexpression of $A t M K K K 18$ led to accelerated leaf senescence, a phenotype usually induced by ABA (Matsuoka et al. 2015). Seed dormancy is usually promoted by ABA (Jin et al. 2018). Since AtMKKK18 has been shown to positively regulate the ABA signal (de Zelicourt et al. 2016), it has been speculated that AtMKKK18 also positively regulates seed dormancy. The results of the present study showed that rice seed dormancy is negatively regulated by $M K K K 62$. This result suggests that the regulatory mechanisms of seed dormancy differ between Arabidopsis thaliana and rice.

In Arabidopsis, Raf10 and Raf11 belong to the RAF subfamily of MKKK genes. Freshly harvested seeds of the mutants raf10 and raf11 without stratification were able to germinate on MS medium. The seed germination rate of the raf10/raf11 double mutant was higher than those of the single mutants, indicating that both Raf10 and Raf11 are required for seed dormancy (Lee et al. 2015). In rice, there are many homologous genes to AtMKKK14/15/16/ 17/18, Raf11 and Raf10. The functions of these genes are still unknown. We believe that further research will reveal more and more of the MKKKs that are involved in the regulation of seed dormancy.

\section{MKKK62-MKK3-MAPK7/14 module regulates seed dormancy} None of the studies in which AtMKKK14/15/16/17/18 were overexpressed in Arabidopsis reported effects on seed dormancy (Choi et al. 2017; Matsuoka et al. 2015), while the overexpression MKKK62 was found to cause

Table 2 Target sites of MKK3 and sequence of target sites in knockout progenies

Target sites of MKK3 and sequence of target sites in knockout progenies

\begin{tabular}{clll}
\hline \multirow{2}{*}{ Code } & $\begin{array}{l}\text { Target site1(MKK3) } \\
\text { CTGTTCGACGACCCGGACAAGGG }\end{array}$ & $-\cdots$ & Target site2 (MKK3) \\
\hline mkk3-1 & CTGTTCGACGACCCGGA----- & --- & ----- TGGTAAGCACGGGCGCG \\
mkk3-2 & CTGTTCGACGACCCGGACCAAGGG & $-\cdots$ & CCTACG-GGTAAGCACGGGCGCG \\
mkk3-3 & CTGTTCGACGACCCGGAA----- & --- & ----- TGGTAAGCACGGGCGCG \\
\hline
\end{tabular}

Note: In Table 2, two target sites were selected from $M K K 3$; PAM region is shown in blue, inserted nucleotides are shown in green, dot indicates the same nucleotide as WT, dash indicates deletion of nucleotide from corresponding site. 
Table 3 Target sites of MAPK7/14 and sequences of target sites in knockout progenies

Target sites of MAPK 7/14 and sequences of target sites in knockout progenies

\begin{tabular}{cll}
\hline Code & Target site (MAPK7) & Target site (MAPK14) \\
& CCGTGTGGAGCACTGAGGACCC & CCATCGGGCGAGAGCTTATGGG \\
\hline mapk7/14-1 & CCGTGTGGGTGCACTGAGGACCC & CCATCGGGGAGGAGTTATGGG \\
mapk7/14-2 & CCGTGT-----CACTGAGGACCC & CCATCGGGGGAGGAGTTATGG \\
mapk7/14-3 & CCGTGT-GATGCACTGAGGACCC & CCATCGGGGGAGGAGTTATGGG \\
\hline
\end{tabular}

Note: In Table 3one target site was selected from MAPK7, and the other from MAPK14.

PAM region is shown in blue, inserted nucleotides are shown in green, dash indicates deletion of nucleotide from corresponding site.

serious PHS in the present study. It is unlikely that AtMKKK18 and MKKK62 have similar functions in the regulation of seed dormancy. In addition, the nomenclature of MAPK proteins is inconsistent, and many protein interactions in MAPK cascade in Arabidopsis need to be re-validated in rice.

To further explore the regulatory mechanism of MKKK62, we used $\mathrm{Y} 2 \mathrm{H}$ experiments to screen for interactions between MKKs proteins and MKKK62 using MKKK62 as the bait. MKK3 and MKK10-2 were screened from seven MKK proteins. In Arabidopsis, similar proteins (AtMKKK14/15/16/17/18) were found to interact with AtMKK3 in $\mathrm{Y} 2 \mathrm{H}$, bimolecular fluorescence complementation, and pull-down analyses (Choi et al. 2017). AtMKKK14/15/16/17/18 were also shown to interact with AtMKK $2 / 4 / 5$, although the interactions were weaker. We did not observe similar results in the present study.

In this study, MAPK7 and MAPK14 interacted with MKK3 (Fig. 4a, d, and e). According to the phylogenetic relationships of Arabidopsis and rice MAPK genes, MAPK7 and MAPK14 belong to Clade C in the MAPK phylogeny (Hamel et al. 2006). In a previous study, all members of $\mathrm{C}$ clade were found to interact with MKK3 in Arabidopsis (Dóczi et al. 2007). The results of our study were consistent with this conclusion. In other study, MKK10-2 was found to interact with MAPK7 (Wankhede et al. 2013), but we did not observe this interaction in our study. It is possible that our screening conditions were more stringent $(200 \mathrm{ng} / \mathrm{mL} \mathrm{AbA}$ in quadruple-dropout medium), and so weak interactions were not detected.

It has been reported that MKK3 can conduct the jasmonic acid signal by activating MAPK6(Sethi et al. 2014; Takahashi et al. 2007). We did not detect any interaction between MKK3 and MAPK6 in our Y2H experiments, suggesting that MAPK6 may be activated indirectly by MKK3. The interaction between MKKK62 and MKK4 predicted in a previous study (Jung et al. 2010) was not detected in our study. These results reflect the complexity of the MAPK signaling system, and highlight the importance of experimentally verifying all interactions in the possible MAPK cascade. At $23 \mathrm{DAH}$, compared with $\mathrm{OE}, \mathrm{OE} / m k k 3(\mathrm{P})$ and $\mathrm{OE} / \operatorname{mapk7}(\mathrm{P}) /$ mapk14(P) re-obtained seed dormancy (Fig. 5a). After maturity, seed dormancy was stronger in $\mathrm{OE} / m k k 3(\mathrm{P})$ and $\mathrm{OE} / \operatorname{mapk} 7(\mathrm{P}) / \operatorname{mapk14}(\mathrm{P})$ than in WT (Fig. 5b, and c). It indicated that MKK3, MAPK7, and MAPK14 participate in dormancy regulation and function downstream of MKKK62. Combined with the $\mathrm{Y} 2 \mathrm{H}$ and pull-down results, these findings implied that the MKKK62-MKK3-MAPK7/14 module regulates seed dormancy and that MKK10-2does not belong to this MAPK module. During the course of our research, $M K K 3$ was found to control seed dormancy in wheat and barley using map-based cloning method (Nakamura et al. 2016; Torada et al. 2016). In this paper, we described in detail each number of the MAPK module that controls seed dormancy in rice.

Decreased $A B A$ sensitivity in $O E$ results in the loss of seed dormancy

The main hormones controlling seed dormancy are ABA and GAs. In Arabidopsis, ABA was shown to increase the expression level of AtMKKK18 (Matsuoka et al. 2015). In this study, the ABA content was higher in OE than in WT (Fig. 2). These results indicate that the MAPK cascade not only responds to the ABA signal, but also influences the ABA level. The OE plants in this study lost seed dormancy. We speculated that the ABA sensitivity of the $\mathrm{OE}$ lines was decreased. The ABA treatment inhibited the shoot and root growth of $\mathrm{OE}$ and WT, but the inhibition was greater in WT than in OE (Fig. 3a, and b). This result was consistent with our speculation that OE lines had decreased ABA sensitivity. In Arabidopsis, the transcription of AtMFT was promoted by ABA (Xi et al. 2010). In our study, the OE lines showed increased ABA content, but decreased transcript levels of OsMFT (Fig. 6), indicating that ABA signal transduction was inhibited in the $\mathrm{OE}$ lines. These 
a

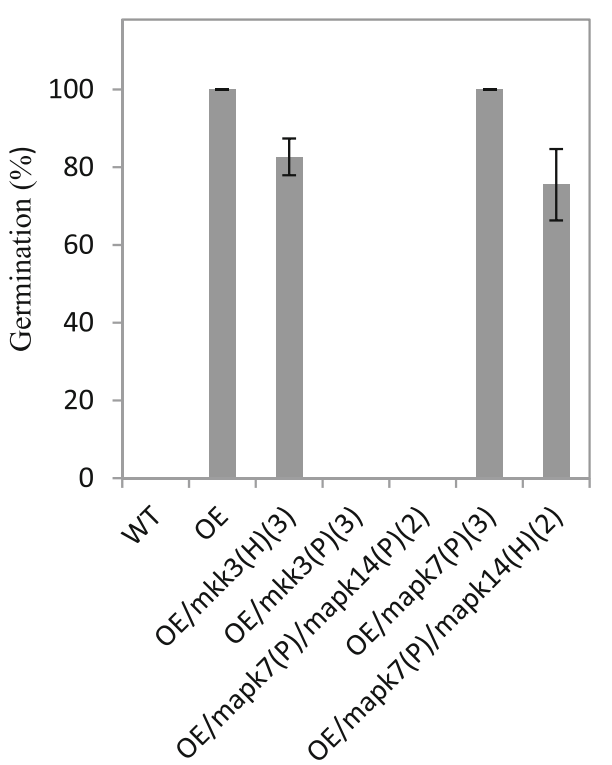

b

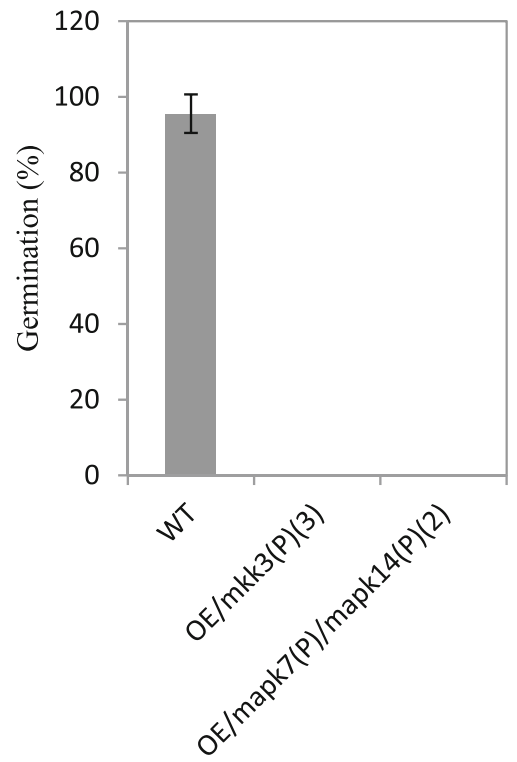

c

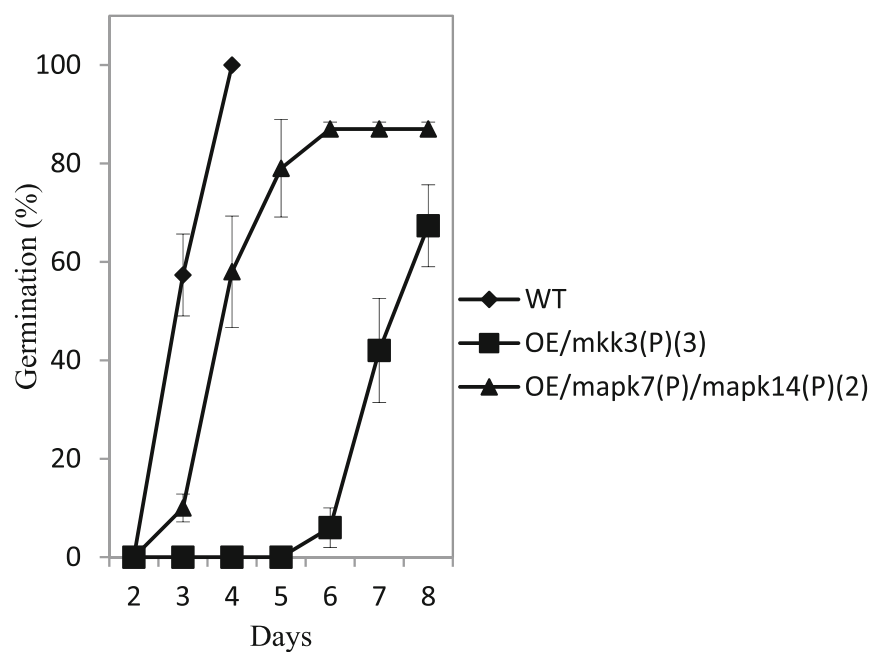

Fig. 5 Germination phenotype of knockout progenies. a, At 23 DAH, seeds were collected from different knockout progenies and germination percentage was calculated at 2 DAl. b, At $30 \mathrm{DAH}$, seeds were collected from homozygous knockout progenies and germination percentage was calculated at 5 DAl. c, Time course of germination of homozygous OE/mkk3 and OE/mapk7/mapk 14 seeds after storage at $37^{\circ} \mathrm{C}$ for 1 month. mkk3, MKK3 knockout plant; mapk7, MAPK7 knockout plant; mapk14, MAPK14 knockout plant; P, homozygous positive; H, heterozygous; Number in parentheses indicates number of knockout lines. Values are mean \pm SD

results also supported that ABA sensitivity was reduced in the OE lines.

The fy-1 mutant in Arabidopsis germinated quickly without stratification or after-ripening, Further analyses showed that the ABA sensitivity of $f y-1$ was lower than that of WT. However, the ABA level was higher in $f y-1$ seeds than in WT seeds, despite the stronger dormancy of WT seeds than $f y-1$ seeds (Jiang et al. 2012). A previous study reported that the Arabidopsis mutants raf10 and raf11 were less sensitive to ABA inhibition of seed germination than was WT, and the overexpression of Raf10 resulted in delayed seed germination and enhanced ABA sensitivity (Lee et al. 2015). All these results suggested that ABA sensitivity is more important than ABA content in germination progress. When seeds of AtMKKK16-OE lines and WT were treated with $\mathrm{ABA}$, the relative germination percentages were higher in the AtMKKK16-OE lines than in WT. However, primary root elongation in the AtMKKK16-OE lines was found to be slightly hypersensitive to $\mathrm{ABA}$ inhibition 


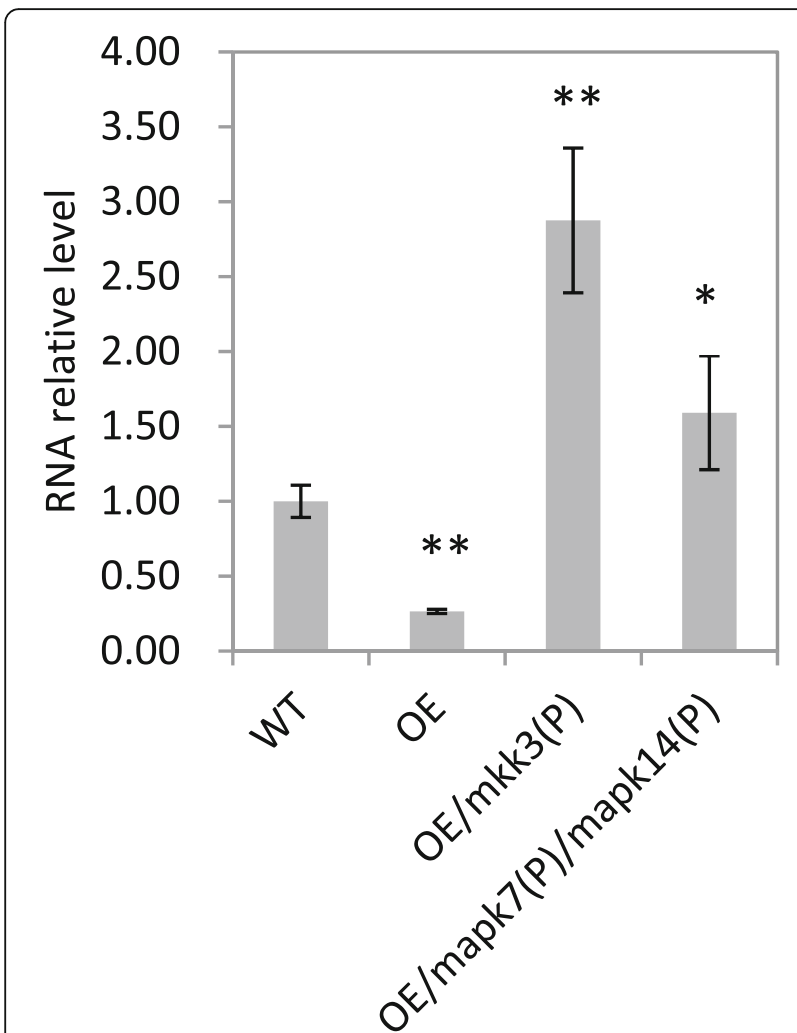

Fig. 6 Relative transcript levels of OsMFT in different transgenic materials. Transcript level of OSMFT was analyzed by real-time PCR using seeds collected at $23 \mathrm{DAH}$. Values shown are the mean $\pm \mathrm{SD}$ of triplicate independent biological samples $(n=3)$. Transcript level was normalized to that of OSMFT in WT. Student's t-tests were used to generate $P$ values

(Choi et al. 2017). These results indicated that the ABA sensitivity of the primary root was not decreased in AtMKKK16-OE lines, which would explain why the seed dormancy was not greatly affected in AtMKKK16-OE.

\section{MKKK62-MKK3-MAPK7/MAPK14 regulates OsMFT to mediate seed dormancy}

$M F T$ encodes a protein in the phosphatidyl ethanolamine-binding protein family. In wheat, the down-regulation of TaMFT under high temperature led to PHS, and the overexpression of TaMFT suppressed germination(Nakamura et al. 2011). The mis-splicing of TaMFT was shown to cause PHS susceptibility (Liu et al. 2015).The results of those studies showed that the transcriptional level of TaMFT controlled seed dormancy. In Arabidopsis and soybean, MFT proteins were also found to promote seed dormancy (Dave et al. 2016; Li et al. 2014; Vaistij et al. 2013). In the present study, the transcript levels of OsMFT were significantly decreased in $\mathrm{OE}$ and significantly increased in $\mathrm{OE} / m k k 3(\mathrm{P})$ and $\mathrm{OE} /$ mapk7(P)/mapk14(P) (Fig. 6). Seed dormancy was also decreased in $\mathrm{OE}$ and increased in $\mathrm{OE} / m k k 3(\mathrm{P})$ and OE/mapk7(P)/mapk14(P) (Fig. 1a, and Fig. 5a, b). Thus, we speculated that the MKKK62-MKK3-MAPK7/ MAPK14 module controls seed dormancy by regulating the transcription of OsMFT (Fig. 7).

\section{Conclusion}

The overexpression of MKKK62 in rice decreased seed dormancy levels and ABA sensitivity at the germination stage. We screened the downstream proteins of MKKK62 by $\mathrm{Y} 2 \mathrm{H}$ and pull-down analyses and clarified their function in dormancy regulation by knockout experiment. These results revealed the entire MAPK module, MKKK62-MKK3-MAPK7/14, that controls seed dormancy in rice. Expression analyses showed that the MKKK62-MKK3-MAPK7/14 module may control seed dormancy by regulating the expression of OsMFT. Further research is required to understand how OsMFT is regulated by MKKK62-MKK3-MAPK7/14 and what relationships exist among ABA, GAs, and the MKKK62-MKK3-MAPK7/14 module.

\section{Methods \\ Plant materials and growth conditions}

The rice cultivar ZH11 and N22 were used in this study. ZH11 is a Japornica rice variety with weak seed dormancy, and N22 is an Indica rice variety with strong seed dormancy(Wan et al. 2005). All materials were grown in the greenhouse of Guangdong Academy of Agricultural Sciences in Guangdong, China.

\section{Germination test}

The panicles or at least 30 rice seeds were dipped in water and then placed in an incubator $\left(30{ }^{\circ} \mathrm{C}\right.$, humidity $>95 \%)$. The germination percentage was calculated at indicated times.

\section{Development of MKKK62-overexpression plants}

The $M K K K 62$ coding sequence (CDS) was amplified using the primers OX-F and OX-R from cDNA from seeds of the rice cultivar $\mathrm{ZH} 11$ (all primers used in this study are listed in Additional file 8: Table S2). The PCR product was

$$
\text { MKKK62 } \stackrel{\mathrm{P}}{\longrightarrow} \text { MKK3 } \stackrel{\mathrm{P})}{\longrightarrow} \text { MPK7/14 } \stackrel{(\mathrm{P})}{\longrightarrow} \quad \longrightarrow \text { OsMFT } \rightarrow \text { Seed dormancy }
$$

Fig. 7 Model of regulation of seed dormancy in rice. Arrows indicate transcriptional activation, flat-ended arrows indicate transcriptional repression. Arrows with $\odot$ indicate activation by phosphorylation. Question mark indicates unknown part 
double-digested with HindIII/BamHI and cloned into the pOX vector to place $M K K K 62$ under the control of the ubiquitin promoter. Hygromycin B phosphotransferase was the selectable marker. The vector was electroporated into Agrobacterium tumefaciens EHA105, and then introduced into the rice varieties ZH11 and N22 via Agrobacterium-mediated genetic transformation.

\section{$\mathrm{Y} 2 \mathrm{H}$ assays}

The Matchmaker Gold Yeast Two-Hybrid System (Clontech, Palo Alto, CA, USA) was used to evaluate protein-protein interactions. We used cDNA obtained from $\mathrm{ZH} 11$ as the template for PCR amplification of MKKK62, OsMAPKs, and OsMKKs, with specific primers containing restriction enzyme recognition sequences. The bait gene was inserted into the pGBKT7 vector, the prey gene was inserted into the pGADT7 vector and the autoactivation activity was tested. These clones were used for $\mathrm{Y} 2 \mathrm{H}$ screening. For yeast transformation, yeast competent cells (Y2HGold) were prepared according to the manufacturer's instructions (PT4084-1, Clontech). Possible interacting constructs were co-transformed into Y2HGold competent cells, and co-transformants were initially screened on double-dropout medium lacking Leu and $\operatorname{Trp}(\mathrm{SD} /-\mathrm{Leu} /-\mathrm{Trp})$. The co-transformed cells were then streaked onto quadruple-dropout medium deficient in Ade, His, Leu, and $\operatorname{Trp}$ (SD/-Ade/-His/-Leu/-Trp). The genes used in these analyses were as follows: rice MKKK62 (LOC_Os01g50420), seven rice MKKs ((LOC_OsO6g27890 (OsMKK3), LOC_Os03g12390 (OsMKK10-2), LOC_Os06g05520 (OsMKK1), LOC_Os01g32660 (OsMKK6), LOC_Os02g54600 (OsMKK4), LOC_Os06g09180 (OsMKK5), and LOC_Os02g46760 (OsMKK10-1)), and 15 rice MAPKs ((LOC_Os02g05480 (OsMAPK14), LOC_Os06g48590 (OsMAPK7), LOC_Os03g17700 (OsMAPK3), LOC_Os10g38950 (OsMAPK4), LOC_Os06g06090 (OsMAPK6), LOC_Os11g17080 (OsMAPK16), LOC_OsO6g49430 (OsMAPK17-1), LOC_Os02g04230 (OsMAPK17-2), LOC_Os01g43910 (OsMAPK20-1), LOC_Os05g50560 (OsMAPK20-2), LOC_Os06g26340 (OsMAPK20-3), LOC_Os01g47530 (OsMAPK20-4), LOC_Os05g49140 (OsMAPK20-5), LOC_Os05g50120 (OsMAPK21-1), and LOC_Os01g45620 (OsMAPK21-2)).

\section{GST pull-down assay}

$M K K K 62$ and MKK3 fragments were cloned into pGEX-4T-1 in frame with the GST tag to generate GST-MKKK62 and GST-MKK3 constructs. MKK3, $M K K 10-2$, MAPK7, and MAPK14 fragments were cloned into the pET-28a $(+)$ vector in frame with the His tag to generate His-MKK3, His-MKK10-2, His-MAPK7, and His-MAPK14 constructs. Details of primers are given in Additional file 8: Table S2. All the constructed vectors were transformed and expressed in E. coli strain BL21. GST-fused proteins were extracted and incubated with Glutathione Sepharose ${ }^{\mathrm{Tu}} 4 \mathrm{~B}$ beads at $4{ }^{\circ} \mathrm{C}$ for $1 \mathrm{~h}$. The beads were collected by centrifugation and washed three times with lysis buffer. His-fused proteins were added and incubated at $4{ }^{\circ} \mathrm{C}$ overnight. The beads were collected and washed three times with lysis buffer. Finally, the proteins were eluted with reduced glutathione, boiled with loading buffer, and analyzed on a $10 \%$ SDS-PAGE gel. Diluted anti-GST antibody and anti-His antibody were used for immunoblotting analysis.

\section{Development of knockout lines}

Target sites were selected for each gene (MKK3, MKK20-1, MAPK7, MAPK14) using tools at the following URL: http://cbi.hzau.edu.cn/crispr/. Two target sites for MKK3 were selected. According to the literature (Ma et al. 2015), the corresponding primers were synthesized and expression cassettes were obtained by overlapping PCR. The expression cassettes were respectively inserted into pYLCRISPR/Cas9Pubi-B to construct the knockout vector MB-MKK3. The knockout vector MB-MKK20-1 was constructed using a similar method. Two target sites (one for MAPK7 and the other for MAPK14) were selected to construct the knockout vector MB-MAPK7/14. Phosphinothricin acetyltransferase was the selectable marker. The vectors were introduced into OE1 via Agrobacterium-mediated genetic transformation. To analyze the transgenic lines, we designed detection primers for each gene to amplify the fragment containing the target sites. The amplification products were sequenced. According to the sequencing results, knockout lines were screened for subsequent experiments.

\section{Measurement of ABA and GAs contents}

The ABA and GAs were extracted from rice seeds as described elsewhere (Pan et al. 2010), and analyzed by liquid chromatography - mass spectrometry (LC-MS/MS). For these analyses, we used a reversed phase ultra-fast liquid chromatograph (Shimadzu, Kyoto, Japan) coupled with a tandem triple quadrupole mass spectrometer (API4000, AB SCIEX, Foster City, CA, USA). The hormone compounds were quantified in multiple reaction monitoring (MRM) mode using the optimized MS/MS conditions listed in Additional file 9: Table S3. The MS conditions were as follows: source, turbo ion spray; ion polarity, negative; ion spray voltage, $-4500 \mathrm{~V}$; source temperature, $550^{\circ}$ C; gas, nitrogen; curtain gas, 30 psi; nebulizing gas (GS1), 55 psi; Collision gas (GS2), 55 psi; scan type, MRM; Q1 resolution: unit; Q3 resolution: unit. Analyst 1.5.2 software (AB SCIEX) was used to control the instrument and to acquire and process all MS data. 


\section{Gene transcript analyses by real-time $P C R$}

To compare the transcriptional levels of selected genes between transgenic and WT plants, seeds or leaves were sampled from three biological replicates at indicated times and immediately frozen in liquid nitrogen. We extracted RNA from samples (each $300 \mathrm{mg}$ ) using Trizol, and $1 \mu \mathrm{g}$ total RNA was reverse-transcribed into cDNAs. Real-time PCR primers were designed based on the Nipponbare genome sequence (Kawahara et al. 2013). The primers and SYBR Premix ExTaq (Takara, Otsu, Japan) were used to amplify the target gene from the cDNA template(Details about the primers are listed in Additional file 8: Table S2). The EF1 $\alpha$ gene $(L O-$ C_Os03g08020) was used as the internal control.

\section{Additional Files}

Additional file 1: Figure S1. Expression pattern of MKKK62. Transcript level of MKKK62 was analyzed by real-time PCR using total RNA isolated from shoots (Sh), leaves (L), and seeds (S). Number following $S$ indicates number of days after heading when seeds were sampled. Transcript level was normalized to that of MKKK62 at $30 \mathrm{DAH}$. Values shown are mean \pm SD of three independent biological samples. (DOCX $17 \mathrm{~kb}$ )

Additional file 2: Figure S2. Germination phenotype of WT and OE lines at $23 \mathrm{DAH}$. Panicle branches were harvested at $23 \mathrm{DAH}$ and kept under germination conditions for 2 days. (DOCX $885 \mathrm{~kb}$ )

Additional file 3: Figure S3. PCR detection of hygromycin gene from OE progenies. M, Marker DL2000; lanes 1-10, samples of non-germinated seeds at $23 \mathrm{DAH}$; lanes 11-20, samples of germinated seeds at $23 \mathrm{DAH}$. (DOCX $147 \mathrm{~kb}$ )

Additional file 4: Figure S4. Germination phenotype of seeds harvested at $23 \mathrm{DAH}$ under ABA treatment. a, Germination phenotype of OE lines after 2 days treatment with ABA; $b$, Effect of ABA concentration on shoot growth of OE lines. OE seeds were harvested at $23 \mathrm{DAH}$ and placed in a 9-cm Petri dish containing filter paper; $10 \mathrm{ml} \mathrm{ABA}$ solution with indicated concentration was added. In mock treatment, water containing an equal volume of solvent was added. Values are mean \pm SD of three OE lines (ten replicates/line). (DOCX $171 \mathrm{~kb}$ )

Additional file 5: Figure S5. Overexpression of MKKK62 decreased seed dormancy of N22. Overexpression vector of MKKK62 was introduced into N22 by Agrobacterium-mediated transformation. Three overexpression lines (N22-OE1, N22-OE2, and N22-OE3) were selected for germination test. In $\mathrm{T}_{0}$ generation, panicles of OE lines were harvested at $30 \mathrm{DAH}$ for germination tests. At $2 \mathrm{DAl}$, most OE seeds germinated while seeds of N22 did not. (DOCX $611 \mathrm{~kb}$ )

Additional file 6: Table S1. Target sites of MKKK62 and sequence results of MKKK62-knockout lines (DOCX $17 \mathrm{~kb}$ )

Additional file 7: Figure S6. Time course of germination of $m k k k 62$ seeds. In the late cropping season in 2017, seeds were harvested at 30 DAH and kept under germination conditions immediately. Germination percentage was scored daily for 5 days. $m k k k 62-1, m k k k 62-2$ and $m k k k 62-3$ are three independent MKKK62-knockout lines. Values shown are mean \pm SD of three replicates. (DOCX $17 \mathrm{~kb}$ )

Additional file 8: Table S2. Primers used in this paper. (DOCX $61 \mathrm{~kb}$ ) Additional file 9: Table S3. Characteristic fragment ions of hormone standards and optimized MS/MS conditions. (DOCX $15 \mathrm{~kb}$ )

\section{Abbreviations}

ABA: Abscisic acid; AbA: Aureobasidin A; DAH: Days after heading; DAl: Days after imbibition; MAPK: Mitogen-activated protein kinase; MKK: Mitogenactivated protein kinase kinase; MKKK: Mitogen-activated protein kinase kinase kinase; OE: Overexpression lines; PHS: Pre-harvest sprouting; WT: Wild type

\section{Acknowledgments}

We thank Liu Yaoguang of South China Agriculture University for providing the plasmids pOX and pYLCRISPR/Cas9Pubi-B. We thank Jennifer Smith, PhD, from Liwen Bianji, Edanz Group China (www.liwenbianji.cn/ac), for editing the English text of a draft of this manuscript.

\section{Funding}

This study was supported by The National Key Research Development Program of China(2016YFD0101102), Guangdong Provincial Natural Science Foundation (2017A030313151), National Natural Science Foundation of China (31471426), Guangdong Science and Technology Project (2015B020231002 and 2017B030314173) and The Team Project of Guangdong Agricultural Department (2017LM2148).

\section{Availability of data and materials}

All data supporting the conclusions of this article are provided in article and additional files (Additional file 1, 2, 3, 4, 5: Figure S1-S6, Table S1-S3).

\section{Authors' contributions}

XXM, JJZ, WGL and FW conceived and designed the experiment. XXM conducted the experiments, performed data analysis, and wrote the manuscript. SJY and JWH analyzed the contents of ABA and GAs. QL, JFD, TFY, JLZ, SHZ, and BL participated in experiments. FW drafted the proposal and corrected the manuscript. All authors read and approved the final manuscript.

\section{Ethics approval and consent to participate}

Not applicable.

\section{Consent for publication}

Not applicable.

\section{Competing interests}

The authors declare that they have no competing interests.

\section{Publisher's Note}

Springer Nature remains neutral with regard to jurisdictional claims in published maps and institutional affiliations.

\section{Author details}

${ }^{1}$ Guangdong Academy of Agricultural Sciences, Rice Research Institute, Guangzhou 510640, China. ${ }^{2}$ Guangdong Key Laboratory of New Technology in Rice Breeding, Guangzhou 510640, China. ${ }^{3}$ Guangdong Key Laboratory for Innovative Development and Utilization of Forest Plant Germplasm, College of Forestry and Landscape Architecture, SCAU, Guangzhou 510642, China. ${ }^{4}$ Agro-biological Gene Research Center, Guangdong Academy of Agricultural Sciences, Guangzhou 510640, China.

Received: 5 July 2018 Accepted: 11 December 2018 Published online: 22 January 2019

\section{References}

Bentsink L, Jowett J, Hanhart CJ, Koornneef M (2006) Cloning of DOG1, a quantitative trait locus controlling seed dormancy in Arabidopsis. Proc Natl Acad Sci 103:17042-17047

Choi S, Lee S, Na Y, Jeung S, Kim SY (2017) Arabidopsis MAP3K16 and other saltinducible MAP3Ks regulate ABA response redundantly. Mol Cells 40:230-242

Chono M, Matsunaka H, Seki M, Fujita M, Kiribuchi-Otobe C, Oda S, Kojima H, Nakamura S (2015) Molecular and genealogical analysis of grain dormancy in Japanese wheat varieties, with specific focus on MOTHER OF FT AND TFL1 on chromosome 3A. Breed Sci 65:103-109

Colcombet J, Hirt H (2008) Arabidopsis MAPKs: a complex signalling network involved in multiple biological processes. Biochem J 413:217-226

Danquah A, Zélicourt A, Boudsocq M, Neubauer J, Nicolas FDF, Leonhardt N, Pateyron S, Gwinner F, Tamby JP, Ortiz-Masia D (2015) Identification and characterization of an ABA-activated MAP kinase cascade in Arabidopsis thaliana. Plant J 82:232

Dave A, Vaistij FE, Gilday AD, Penfield SD, Graham IA (2016) Regulation of Arabidopsis thaliana seed dormancy and germination by 12-oxophytodienoic acid. J Exp Bot 67:2277-2284 
de Zelicourt A, Colcombet J, Hirt H (2016) The role of MAPK modules and ABA during abiotic stress signaling. Trends Plant Sci 21:677-685

Dóczi R, Brader G, Pettkószandtner A, Rajh I, Djamei A, Pitzschke A, Teige M, Hirt $H$ (2007) The Arabidopsis mitogen-activated protein kinase kinase MKK3 is upstream of group C mitogen-activated protein kinases and participates in pathogen signaling. Plant Cell 19:3266-3279

Fang J, Chu C (2008) Abscisic acid and the pre-harvest sprouting in cereals. Plant Signal Behav 3:1046-1048

Finch-Savage WE, Leubner-Metzger G (2006) Seed dormancy and the control of germination. New Phytol 171:501-523

Finkelstein R, Reeves W, Ariizumi T, Steber C (2008) Molecular aspects of seed dormancy. Annu Rev Plant Biol 59:387-415

Footitt S, Douterelo-Soler I, Clay H, Finch-Savage WE (2011) Dormancy cycling in Arabidopsis seeds is controlled by seasonally distinct hormone-signaling pathways. Proc Natl Acad Sci 108:20236-20241

Footitt S, Huang Z, Clay HA, Mead A, Finch-Savage WE (2013) Temperature, light and nitrate sensing coordinate Arabidopsis seed dormancy cycling, resulting in winter and summer annual phenotypes. Plant J 74:1003-1015

Graeber K, Linkies A, Steinbrecher T, Mummenhoff K, Tarkowska D, Tureckova V, Ignatz M, Sperber K, Voegele A, de Jong H, Urbanova T, Strnad M, LeubnerMetzger G (2014) DELAY OF GERMINATION 1 mediates a conserved coatdormancy mechanism for the temperature- and gibberellin-dependent control of seed germination. Proc Natl Acad Sci 111:E3571-E3580

Graeber K, Nakabayashi K, Miatton E, Leubner-Metzger G, Soppe WJ (2012) Molecular mechanisms of seed dormancy. Plant Cell Environ 35:1769-1786

Group. M (2002) Mitogen-activated protein kinase cascades in plants: a new nomenclature. Trends Plant Sci 7:301-308

Gu X, Foley ME, Horvath DP, Anderson JV, Feng J, Zhang L, Mowry CR, Ye H, Suttle JC, Kadowaki K-i, Chen Z (2011) Association between seed dormancy and pericarp color is controlled by a pleiotropic gene that regulates abscisic acid and flavonoid synthesis in weedy red rice. Genetics 189:1515-1524

Hamel LP, Nicole MC, Sritubtim S, Morency MJ, Ellis M, Ehlting J, Beaudoin N, Barbazuk B, Klessig D, Lee J, Martin G, Mundy J, Ohashi Y, Scheel D, Sheen J, Xing T, Zhang S, Seguin A, Ellis BE (2006) Ancient signals: comparative genomics of plant MAPK and MAPKK gene families. Trends Plant Sci 11:192-198

Hattori T, Terada T, Hamasuna ST (1994) Sequence and functional analyses of the rice gene homologous to the maize Vp1. Plant Mol Biol 24:805-810

Holdsworth MJ, Bentsink L, Soppe WJ (2008) Molecular networks regulating Arabidopsis seed maturation, after-ripening, dormancy and germination. New Phytol 179:33-54

Jiang S, Kumar S, Eu YJ, Jami SK, Stasolla C, Hill RD (2012) The Arabidopsis mutant, fy-1, has an ABA-insensitive germination phenotype. J Exp Bot 63:2693-2703

Jin D, Wu M, Li B, Bucker B, Keil P, Zhang S, Li J, Kang D, Liu J, Dong J, Deng XW, Irish V, Wei N (2018) The COP9 signalosome regulates seed germination by facilitating protein degradation of RGL2 and ABI5. PLOS Genet 14:e1007237

Jung KH, Cao P, Seo YS, Dardick C, Ronald PC (2010) The Rice kinase Phylogenomics database: a guide for systematic analysis of the rice kinase super-family. Trends Plant Sci 15(11):595-599

Kawahara Y, Bastide MDL, Hamilton JP, Kanamori H, Mccombie WR, Shu O, Schwartz DC, Tanaka T, Wu J, Zhou S (2013) Improvement of the Oryza sativa Nipponbare reference genome using next generation sequence and optical map data. Rice 6:1-10

Lampard GR, Lukowitz W, Ellis BE, Bergmann DC (2009) Novel and expanded roles for MAPK signaling in Arabidopsis stomatal cell fate revealed by cell type-specific manipulations. Plant Cell 21:3506-3517

Lee SJ, Lee MH, Kim JI, Kim SY (2015) Arabidopsis putative MAP kinase kinase kinases Raf10 and Raf11 are positive regulators of seed dormancy and ABA response. Plant Cell Physiol 56:84-97

Leonhardt N, Kwak JM, Robert N, Waner D, Leonhardt G, Schroeder Jl (2004) Microarray expression analyses of Arabidopsis guard cells and isolation of a recessive abscisic acid hypersensitive protein phosphatase $2 C$ mutant. Plant Cell 16:596-615

Li C, Ni P, Francki M, Hunter A, Zhang Y, Schibeci D, Li H, Tarr A, Wang J, Cakir M, Yu J, Bellgard M, Lance R, Appels R (2004) Genes controlling seed dormancy and pre-harvest sprouting in a rice-wheat-barley comparison. Funct Integr Genomics 4:84-93

Li K, Yang F, Zhang G, Song S, Li Y, Ren D, Miao Y, Song CP (2017) AlK1, a mitogen-activated protein kinase, modulates abscisic acid responses through the MKK5-MPK6 kinase cascade. Plant Physiol 173:1391-1408

Li Q, Fan C, Zhang X, Wang X, Wu F, Hu R, Fu Y (2014) Identification of a soybean MOTHER OF FT AND TFL1 homolog involved in regulation of seed germination. PLoS One 9:e99642
Liu S, Sehgal SK, Lin M, Li J, Trick HN, Gill BS, Bai G (2015) Independent missplicing mutations in TaPHS1 causing loss of preharvest sprouting (PHS) resistance during wheat domestication. New Phytol 208:928-935

Lopez-Molina L, Mongrand S, Chua NH (2001) A postgermination developmental arrest checkpoint is mediated by abscisic acid and requires the ABI5 transcription factor in Arabidopsis. Proc Natl Acad Sci 98:4782-4787

Ma X, Zhang Q, Zhu Q, Liu W, Chen Y, Qiu R, Wang B, Yang Z, Li H, Lin Y, Xie Y, Shen R, Chen S, Wang Z, Chen Y, Guo J, Chen L, Zhao X, Dong Z, Liu Y-G (2015) A robust CRISPR/Cas9 system for convenient, high-efficiency multiplex genome editing in monocot and dicot plants. Mol Plant 8:1274-1284

Matsuoka D, Yasufuku T, Furuya T, Nanmori T (2015) An abscisic acid inducible Arabidopsis MAPKKK, MAPKKK18 regulates leaf senescence via its kinase activity. Plant Mol Biol 87:565-575

Nakamura S, Abe F, Kawahigashi H, Nakazono K, Tagiri A, Matsumoto T, Utsugi S, Ogawa T, Handa H, Ishida H, Mori M, Kawaura K, Ogihara Y, Miura H (2011) A wheat homolog of MOTHER OF FT AND TFL1 acts in the regulation of germination. Plant Cell 23:3215-3229

Nakamura S, Pourkheirandish M, Morishige $H$, Kubo Y, Nakamura M, Ichimura K, Seo S, Kanamori H, Wu J, Ando T, Hensel G, Sameri M, Stein N, Sato K, Matsumoto T, Yano M, Komatsuda T (2016) Mitogen-activated protein kinase kinase 3 regulates seed dormancy in barley. Curr Biol 26:775-781

Pan X, Welti R, Wang X (2010) Quantitative analysis of major plant hormones in crude plant extracts by high-performance liquid chromatography-mass spectrometry. Nat Protoc 5:986-992

Rao KP, Richa T, Kumar K, Raghuram B, Sinha AK (2010) In silico analysis reveals 75 members of mitogen-activated protein kinase kinase kinase gene family in rice. DNA Res 17:139-153

Sato Y, Takehisa H, Kamatsuki K, Minami H, Namiki N, Ikawa H, Ohyanagi H, Sugimoto K, Antonio BA, Nagamura Y (2013) RiceXPro version 3.0: expanding the informatics resource for rice transcriptome. Nucleic Acids Res 41:D1206-D1213

Sethi V, Raghuram B, Sinha AK, Chattopadhyay S (2014) A mitogen-activated protein kinase cascade module, MKK3-MPK6 and MYC2, is involved in blue light-mediated seedling development in Arabidopsis. Plant Cell 26:3343-3357

Shu K, Liu XD, Xie Q, He ZH (2016) Two faces of one seed: hormonal regulation of dormancy and germination. Mol Plant 9:34-45

Simsek S, Ohm JB, Lu H, Rugg M, Berzonsky W, Alamri MS, Mergoum M (2014) Effect of pre-harvest sprouting on physicochemical properties of starch in wheat. Foods 3:194-207

Sreenivasulu N, Usadel B, Winter A, Radchuk V, Scholz U, Stein N, Weschke W, Strickert M, Close TJ, Stitt M, Graner A, Wobus U (2008) Barley grain maturation and germination: metabolic pathway and regulatory network commonalities and differences highlighted by new MapMan/PageMan profiling tools. Plant Physiol 146:1738-1758

Sugimoto K, Takeuchi Y, Ebana K, Miyao A, Hirochika H, Hara N, Ishiyama K, Kobayashi M, Ban Y, Hattori T, Yano M (2010) Molecular cloning of Sdr4, a regulator involved in seed dormancy and domestication of rice. Proc Natl Acad Sci 107:5792-5797

Takahashi F, Yoshida R, Ichimura K, Mizoguchi T, Seo S, Yonezawa M, Maruyama K, Yamaguchishinozaki K, Shinozaki K (2007) The mitogen-activated protein kinase cascade MKK3-MPK6 is an important part of the jasmonate signal transduction pathway in Arabidopsis. Plant Cell 19:805-818

Tao YB, Luo L, He LL, Ni J, Xu ZF (2014) A promoter analysis of MOTHER OF FT AND TFL 11 (JCMFT1), a seed-preferential gene from the biofuel plant Jatropha curcas. J Plant Res 127:513-524

Teige M, Scheikl E, Eulgem T, Doczi R, Ichimura K, Shinozaki K, Dangl JL, Hirt H (2004) The MKK2 pathway mediates cold and salt stress signaling in Arabidopsis. Mol Cell 15:141-152

Torada A, Koike M, Ogawa T, Takenouchi Y, Tadamura K, Wu J, Matsumoto T, Kawaura K, Ogihara Y (2016) A causal gene for seed dormancy on wheat chromosome 4A encodes a MAP kinase kinase. Curr Biol 26:782-787

Vaistij FE, Gan Y, Penfield S, Gilday AD, Dave A, He Z, Josse EM, Choi G, Halliday KJ, Graham IA (2013) Differential control of seed primary dormancy in Arabidopsis ecotypes by the transcription factor SPATULA. Proc Natl Acad Sci 110:10866-10871

Wan JM, Cao YJ, Wang CM, Ikehashi H (2005) Quantitative trait loci associated with seed dormancy in Rice. Crop Sci 45:712-716

Wan JM, Jiang L, Tang JY, Wang CM, Hou MY, Jing W, Zhang LX (2006) Genetic dissection of the seed dormancy trait in cultivated rice (Oryza sativa L.). Plant Sci 170:786-792

Wan Y, Poole RL, Huttly AK, Toscano-Underwood C, Feeney K, Welham S, Gooding MJ, Mills C, Edwards KJ, Shewry PR, Mitchell RA (2008) 
Transcriptome analysis of grain development in hexaploid wheat. BMC Genomics 9:121-136

Wankhede DP, Misra M, Singh P, Sinha AK (2013) Rice mitogen activated protein kinase kinase and mitogen activated protein kinase interaction network revealed by insilico docking and yeast two-hybrid approaches. PLoS One 8:e65011

Xi W, Liu C, Hou X, Yu H (2010) MOTHER OF FT AND TFL1 regulates seed germination through a negative feedback loop modulating ABA signaling in Arabidopsis. Plant Cell 22:1733-1748

Xing Y, Jia W, Zhang J (2008) AtMKK1 mediates ABA-induced CAT1 expression and $\mathrm{H}_{2} \mathrm{O}_{2}$ production via AtMPK6-coupled signaling in Arabidopsis. Plant $J$ Cell Mol Biol 54:440-451

Xing Y, Jia W, Zhang J (2009) AtMKK1 and AtMPK6 are involved in abscisic acid and sugar signaling in Arabidopsis seed germination. Plant Mol Biol 70:725-736

Ye H, Feng J, Zhang L, Zhang J, Mispan MS, Cao Z, Beighley DH, Yang J, Gu XY (2015a) Map-based cloning of Seed Dormancy $1-2$ identified a gibberellin synthesis gene regulating the development of endosperm-imposed dormancy in rice. Plant Physiol 169:2152-2165

Ye L, Li L, Wang L, Wang S, Li S, Du J, Zhang S, Shou H (2015b) MPK3/MPK6 are involved in iron deficiency-induced ethylene production in Arabidopsis, Front Plant Sci 6:953

Yoo S-D, Cho Y-H, Tena G, Xiong Y, Sheen J (2008) Dual control of nuclear EIN3 by bifurcate MAPK cascades in $\mathrm{C} 2 \mathrm{H} 4$ signalling. Nature 451:789-795

\section{Submit your manuscript to a SpringerOpen ${ }^{\circ}$ journal and benefit from:}

- Convenient online submission

- Rigorous peer review

- Open access: articles freely available online

High visibility within the field

- Retaining the copyright to your article

Submit your next manuscript at $\boldsymbol{\nabla}$ springeropen.com 